\title{
Analysing the Relationship between Learning Styles and Cognitive Traits*
}

\author{
Sabine Graf ${ }^{1}$, Taiyu Lin $^{2}$, Kinshuk ${ }^{3}$ \\ ${ }^{1}$ Vienna University of Technology, Women's Postgraduate College for Internet Technologies, \\ Austria, sabine.graf@ieee.org \\ ${ }^{2}$ Massey University, Department of Information Systems, New Zealand, taiyu.lin@gmail.com \\ ${ }^{3}$ Athabasca University, School of Computing and Information Systems, Canada, \\ kinshuk@ieee.org
}

\begin{abstract}
The need to provide more holistic adaptivity to students has brought us to investigate the relationship between learning styles and working memory capacity (WMC). The aim of this investigation is to study the relationship between learning styles and WMC in order to get additional information about the students. This information can be used to make more holistic adaptivity possible by improving the student modelling process of both learning styles and WMC. An experiment with 297 participants was conducted. Findings suggest that relationships from WMC to the active/reflective, the sensing/intuitive, and the visual/verbal learning styles exist, whereas the suggested relationship from WMC to sequential/global learning styles could not be found.
\end{abstract}

\section{Introduction}

Providing courses that fit the needs of learners makes learning easier for students. In recent years, researchers focused on the impact of individual characteristics such as learning styles and cognitive traits in technology enhanced learning and how such differences can be incorporated in order to provide adaptive courses.

To be able to provide proper adaptivity, the needs of students have to be known first. With respect to technology enhanced learning, Brusilovsky [1] distinguished between two different ways for getting information about the learners' needs: the collaborative and the automatic student modelling approach. In the former, the students explicitly provide information about themselves (e.g. filling out a

\footnotetext{
* This research has been funded partly by the Austrian Federal Ministry for Education, Science, and Culture, the European Social Fund (ESF) under grant 31.963/46-VII/9/2002.
}

questionnaire), whereas in the latter, the system infers the needs from the behaviour and actions of the students automatically while they are working/learning in the system. The automatic approach is direct and is free from the problem of inaccurate self-conceptions of students. However, a problem with the automatic approach is to get enough reliable information to build a robust student model. As a solution, Brusilovsky [1] recommended the use of additional sources of information. Hence, it is beneficial to find mechanisms that use whatever information about the learner is already available to get as much reliable information to build a more robust student model.

In this paper, we investigate the relationship between learning styles, in particular the FelderSilverman learning style model (FSLSM) [2], and working memory capacity (WMC). WMC is one of the cognitive traits included in the Cognitive Trait Model (CTM) [3, 4], a model to profile students' cognitive traits using student behaviour. The found relationship can then be used to strengthen the inference procedure of student models such as the CTM by including additional information from learning styles. Similarly, the findings can also be used to strength a learning style model which is built not from a questionnaire but from student behaviour observations.

Section 2 presents a brief introduction to FSLSM and WMC. It also lists a set of hypotheses based on our previous literature study. Section 3 describes the study design and Section 4 presents the method used in the experiment. The results are discussed in Section 5 and Section 6 concludes this study.

\section{Background}

Several different learning style models exist in the literature. Looking at adaptive educational systems which incorporate learning styles, FSLSM is one of the most often used model in recent times and some 
researchers even argue that it is the most appropriate model $[5,6]$.

According to FSLSM, each learner is characterized according to four dimensions: active learners learn by trying things out and working with others, whereas reflective learners learn by thinking things through and working alone. Sensing learners like to learn concrete material and tend to be practical, whereas intuitive learners prefer to learn abstract material such as theories and their meanings and tend to be more innovative than sensing learners. Visual learners remember best what they have seen, whereas verbal learners get more out of words, regardless whether they are spoken or written. Sequential learners learn in linear steps and prefer to follow linear stepwise paths, whereas global learners learn in large leaps and are characterized as holistic.

Regarding cognitive traits, working memory capacity is an important factor for learning. In earlier times, working memory was also referred to as shortterm memory. Richards-Ward [7] named it the ShortTerm Store (STS) to emphasise its role of temporal storage of recently perceived information. Working memory allows us to keep active a limited amount of information (roughly $7+-2$ items) for a brief period of time [8]. WMC is included in the Cognitive Trait Model [3, 4], a student model that aims at profiling learners according to their cognitive traits in adaptive web-based educational systems.

A comprehensive literature review [9] investigated the relationship of FSLSM and WMC by looking at studies that deal with the interaction of learning styles, cognitive styles, and cognitive traits. From these studies, direct and indirect relationships between the dimensions of FSLSM and WMC were concluded. As a result from the literature review, it can be summarized that learners with high WMC tend to prefer a reflective, intuitive, and sequential learning style. On the other hand, learners with a low WMC tend to have a more active, sensing, and global learning style. For the visual/verbal dimension, it was identified only an one-directional relationship rather than a bidirectional correlation as for the other dimensions. Therefore, learners with a low WMC tend to prefer a visual learning style but learners with a high WMC might have visual or verbal preferences. On the other hand, learners with a verbal learning style tend to have a high WMC but learners with a visual learning style might have a high or low WMC.

\section{Materials}

The aim of this study is to analyse the relationship between working memory capacity and learning styles by the use of real data and compare it with the results from the literature review. Therefore, we conducted an experiment with 297 students from Vienna University of Technology in Austria. All students were asked to fill out a questionnaire for identifying their learning styles and perform a test to detect their WMC.

In order to identify learning styles, we used the Index of Learning Styles questionnaire (ILS) [10], which is developed to detect learning styles based on the FSLSM. The ILS is an often used instrument and consists of 44 questions which can be answered online. As mentioned earlier, according to FSLSM each learner has a personal preference for each of the four dimensions. In ILS, these preferences are expressed with values between +11 to -11 per dimension, where for instance +11 indicates a strong active preference and -11 a strong reflective preference.

For detecting WMC, we developed Web-OSPAN [11]. Web-OSPAN is a web-based version of the operation word span task (OSPAN) [12]. According to De Neys et al. [13], the operation word span task has become one of the most popular tasks to measure WMC. In the task, subjects are required to perform simple arithmetical operations such as $(2 * 3)+4=10$ and answer whether this operation is true or false. After each operation, a word is presented. The subjects are asked to perform 2-6 arithmetic operations and at the end are asked to recall the words presented after each operation in the correct order. As proposed by Turner and Engle [12], the total number of correct calculations (referred to as process measure, ranged from $0-60$ ), the total number of correct recalled words (referred to as WMC values, ranged from 0-60), the maximum set size the subject had the words recalled correctly (referred to as set size memory span, ranged from 0-6), and the mean response latency are recorded and the total number of correctly recalled words is used as a measure of WMC. Web-OSPAN follows OSPAN [12] in recording these measures. Additionally, WebOSPAN records a partial correct memory span (ranged from 0-60), which counts words as correct even when the order of words is not correct.

\section{Method for statistical data analysis}

Data of students who had more than 15 mistakes in the calculations of Web-OSPAN or spent less than 5 minutes in ILS were discarded because they do not meet the experiment requirements. Data from 225 students were finally used for analyses.

Furthermore, we improved the reliability of ILS (measured by Cronbach's alpha) through removing weak reliable questions ( 1 from the active/reflective dimension, 1 from the sensing/intuitive dimension, 3 
from the visual/verbal dimension, and 2 from the sequential/global dimension).

Data analysis was done by a general and an in-depth analysis. In both, outliers were excluded for the analysed dimension. General analysis dealt with correlation analysis between WMC and ILS dimensions by using rank correlation. Additionally, we analysed the recorded measures gathered from WebOSPAN by correlating them with the WMC values in order to show how significant they are related to WMC. According to the structure of analysed values, Pearson's correlation or rank correlation was applied.

For the in-depth analysis, learning style values were divided into three groups, distinguishing e.g. between an active, balanced, and reflective preference. The groups were built based on recommendations by Felder and colleagues [10, 14] and with respect to the performed reduction of questions for increasing reliability. Consequently, values $>=+4$ indicate a preference for one pole, values $<=-4$ indicate a preference for the other pole and values between +3 and -3 indicate a balanced learning style.

Then, chi-square test was used to identify differences between the groups. If significant differences were detected, further analyses were performed to identify the kind of relation between the groups. These further analyses included correlation analysis between WMC values and the absolute values of ILS in order to identify a correlation between WMC and the strength of preference. Moreover, we split the dataset into two sub-datasets $S_{x}$ and $S_{y}$ in in-depth analysis. $S_{x}$ covers only data representing a balanced preference or a preference for the positive pole, and $S_{y}$ covers only data with an ILS value representing a balanced preference or a preference of the negative pole. For each sub-dataset, correlation analysis and group comparison methods (grouping WMC values and grouping learning style values) were performed.

For the visual/verbal dimension, literature [9] argued for a one-directional rather than a bidirectional relationship. In order to prove one-directional relationships, we separated data into two sub-datasets $F_{x}$ and $F_{y}$, where $F_{x}$ includes only data from verbal learners and $\mathrm{F}_{\mathrm{y}}$ includes only data from visual learners. Then, for each sub-dataset, the number of learners in WMC groups (grouped by steps of 5) was calculated and rank correlation analysis was performed in order to find a correlation between the frequencies of learners with e.g. verbal learning style $\left(F_{x}\right)$ and their WMC. Afterwards, results for $F_{x}$ and $F_{y}$ were compared. The same was done for the two sub-datasets including learners with only high and only low WMC. Due to the high difference in variance in the variables, Kendall's tau can be considered as more robust and is therefore applied for these analyses.

\section{Results}

In the following subsections, we introduce and discuss the results of the conduced analyses for the measures of Web-OSPAN as well as for each learning style dimension.

\subsection{Measures of Web-OSPAN}

The conducted correlation analysis, calculated by Pearson's r, Kendall's tau or Spearman's rho respectively, shows that all other measures gathered from Web-OSPAN are highly significant $(\mathrm{p}<0.001)$ correlated with the WMC values. The set size memory span (tau $=0.649$, rho $=0.757$ ) and the partial correct memory span (tau=0.741, rho=0.883) show a strong positive correlation to the WMC values. Interesting is that the mean response time is negative correlated $(\mathrm{r}=-0.361)$, which indicates that learners who answered quickly answered correctly more often. The values of the process measure show only a low positive correlation (tau $=0.191$, rho $=0.258)$.

\subsection{Active/reflective dimension}

In the general analysis, no significant correlations were found between WMC and the active/reflective values. However, according to the in-depth analysis, the significant result of the chi-square test $\left(\chi^{2}=7.889\right.$, $\mathrm{p}=0.019$ ) indicated that the three groups (active, balanced, and reflective) were different to each other. We found a highly significant negative correlation between the absolute active/reflective values and the WMC values (tau $=-0.169, \mathrm{p}=0.001$; rho $=-0.222$, $\mathrm{p}=0.001$ ), the set size memory span (tau=-0.140, $\mathrm{p}=0.015$; rho $=-0.161, \mathrm{p}=0.015$ ), and the partial correct memory span (tau=-0.167, $\mathrm{p}=0.002$; rho=-0.216, 0.003). These correlations show that learners with a balanced learning style tend to have high WMC, whereas learners with either a very active or a very reflective learning style tend to have low WMC. This hypothesis is furthermore supported by the result of the analysis of the sub-dataset $\mathrm{S}_{\text {act/bal }}$ and $\mathrm{S}_{\text {ref/bal }}$.

Looking at the sub-dataset $\mathrm{S}_{\text {act/bal }}$, which includes only data indicating an active or balanced preference, the correlation analysis resulted in a negative significant correlation between the active/balanced values and WMC values (tau=-0.173, $\mathrm{p}=0.002$; rho=-0.226, $\mathrm{p}=0.003)$, set size memory span (tau=-0.162, $\mathrm{p}=0.014$; rho=-0.191, $\mathrm{p}=0.013$ ), partial correct memory span (tau=-0.142, $\mathrm{p}=0.022$; rho=-0.188, $\mathrm{p}=0.023)$, and process measure (tau=-0.138, $\mathrm{p}=0.019$; rho=-0.177, $\mathrm{p}=0.021$ ). These correlations show that active learners tend to have low 
WMC and balanced learners tend to have high WMC (and vice versa). This is further supported by a significant result of the Mann-Whitney U test ( $\mathrm{U}=2324.5, \mathrm{p}=0.008$ ), comparing the high WMC group and low WMC group over the active/balanced values and indicating that learners with low WMC have a significantly more active learning style than learners with high WMC.

On the other hand, looking at $S_{\text {ref/bal, the }}$ reflective/balanced part of data, we found only a low significant, positive correlation between the WMC values and the reflective/balanced values according to Spearman's rho (rho=0.163, $\mathrm{p}=0.045$ ). However, this relation is supported by the highly significant result of the t-test ( $\mathrm{T}=-3.094, \mathrm{p}=0.002)$, comparing the reflective and balanced group over the WMC values and indicating that reflective learners have significantly lower WMC than balanced learners.

From all these evidences, we can conclude that there exists a significant relationship between the active/reflective dimension and WMC. This relationship shows that the more balanced the learning style is, the higher WMC the learners tend to have. On the other hand, the stronger the preference for either an active or a reflective learning style is, the lower WMC the learners tend to have. Regarding an active learning preference, our results are in agreement with the conclusions from literature [9], since both associate low WMC with an active learning preference. However, regarding a reflective preference, conclusions from literature argued for high WMC. According to our results, active and reflective preferences are associated with low WMC, whereas a balanced learning style is related to high WMC.

\subsection{Sensing/intuitive dimension}

The results of the correlation analysis of the sensing/intuitive and WMC values show a significant negative correlation between sensing/intuitive values and the size set memory span (tau=-0.113, $\mathrm{p}=0.046$; rho=-0.137, $\mathrm{p}=0.045$ ). This result gives an indication for an indirect relationship between WMC and the sensing/intuitive dimension since the WMC values are highly correlated with the size set memory span (as shown in Section 5.1.). The results of the chi-square test $\left(\chi^{2}=8.628, p=0.013\right)$ show that the three groups (sensing, balanced, intuitive) are significantly different from each other. Since the correlation of WMC values and absolute sensing/intuitive values is not significant, this is another indication for a linear correlation between a sensing/intuitive preference and WMC.

Looking at the sub-dataset $\mathrm{S}_{\text {sen/bal }}$, we found a significant negative correlation between the sensing/balanced values and the set size memory span (tau=-0.132, $\mathrm{p}=0.041$; rho=-0.157, $\mathrm{p}=0.041$ ), which again indicates an indirect relation to WMC. Accordingly, low WMC is associated with a sensing learning style and high WMC is associated with a balanced learning style. This is also supported by the results of the group comparison in both directions. The highly significant result $(U=2263, p=0.005)$ from the Mann-Whitney U test between groups of WMC shows that learners with low WMC tend to have a significantly higher preference for a sensing learning style than learners with high WMC. Looking in the other direction, the conducted t-test $(\mathrm{T}=-1.976, \mathrm{p}<0.05)$ shows that learners with a sensing learning style tend to have significantly lower WMC than learners with a balanced learning style.

Considering the intuitive/balanced part, we found only a low, negative correlation between the intuitive/balanced values and the mean response latency (tau=-0.149, $\mathrm{p}=0.032$; rho=-0.205, $\mathrm{p}=0.029$ ). According to the analysis in Section 5.1., only a weak correlation exists between the WMC values and the mean response latency, which seems to be not reliable enough to conclude for an indirect relationship. Also from group comparison, no significant relations were found.

From these results, we can conclude that a sensing learning style is associated with low WMC and the more balanced the learning style becomes, the higher is the tendency to have high WMC. This is in agreement with the conclusions from literature [9]. Furthermore, according to literature this increase of tendency for high WMC continues, the more intuitive the learning style preference becomes. For this second part of the relationship, we found no evidence in the data. This might be reasoned from the few learners with strong intuitive preference (ILS value $<=-8, n=7$ ).

\subsection{Visual/verbal dimension}

As indicated by the literature review [9], we did not find any significant result for a bidirectional relationship between WMC and the visual/verbal dimension, neither with general analysis nor with indepth analysis. Since according to literature an onedirectional relationship was detected, we focused on proving one-directional relationships by using correlation of frequencies in sub-datasets.

Looking at two datasets separating learners with high and low WMC, correlation between frequencies and visual/verbal preferences shows a highly significant and strong positive correlation for both, low WMC learners (tau=0.857, $\mathrm{p}=0.002$ ) and high WMC learners (tau=0.889, $\mathrm{p}=0.001$ ). This was expected since it is know from other studies, summarized in [14], and 
can also be seen from our study that, in general, more learners have a visual than a verbal learning style.

When separating learners with visual and verbal learning preference, correlation analysis of frequencies shows a significant correlation for learners with a verbal learning style (tau=0.51, $\mathrm{p}=0.033$ ). This indicates that in the group of verbal learners, a high frequency is associated with high WMC, whereas few verbal learners have low WMC. In contrast, when looking at learners with a visual style, the result of the correlation is not significant and correlation is lower than for the verbal learners ( $\operatorname{tau}=0.455, \mathrm{p}=0.520$ ).

As a conclusion, our findings are in agreement with the results from the literature review [9].

\subsection{Sequential/global dimension}

According to literature, evidence exists for a relationship between a sequential learning style preference and high WMC as well as a global learning style preference and low WMC [9]. Based on the data of this study, we did not find any evidence that yields to this conclusion. Neither general analysis nor indepth analysis resulted in a significant relationship. Therefore, our findings are in disagreement with literature, by indicating that there exists no relationship between the sequential/global dimension and WMC.

\section{Conclusion and future work}

In this paper, we investigated the relationship between the FSLSM and WMC by conducting an experiment with 297 participants. The results showed a relationship for the active/reflective, the sensing/intuitive, and the visual/verbal dimension, whereas no relationship was found for the sequential/global dimension.

The identified relationships provide additional information about the learners. This additional information can be used in systems that consider only either learning styles or cognitive traits to extend the student model. This leads to more holistic adaptivity by including both learning styles and WMC. Furthermore, the additional information from the relationship can be used to improve the detection of learning styles and/or cognitive traits. This strengthens the existing student models and leads to a better support for holistic adaptivity.

Future work will deal with a more granular analysis by considering specific characteristics within the FSLSM dimensions and looking at their relationships to WMC. Furthermore, we plan to incorporate the findings of this study into the CTM in order to improve the inference process of WMC. We also plan to build a learning style student model using the additional information about cognitive traits in order to improve the automatic student modelling approach.

\section{References}

[1] P. Brusilovsky, Methods and techniques of adaptive hypermedia, User Modeling and User-Adapted Interaction, 6, 1996, pp. 87-129.

[2] R. M. Felder and L. K. Silverman, Learning and teaching styles in engineering education, Engineering Education, 78, 1988, pp. 674-681. Preceded by a preface in 2002: http://www.ncsu.edu/felderpublic/Papers/LS-1988.pdf (retrieved July 23, 2006).

[3] Kinshuk and T. Lin, Cognitive profiling towards formal adaptive technologies in web-based learning communities, International Journal of WWW-based Communities, 1, 2004, pp. 103-108.

[4] T. Lin and Kinshuk, Cognitive profiling in life-long learning, Encyclopedia of International Computer-Based Learning, Idea Group Inc., Hershey, PA, USA, 2005, pp. 245-255.

[5] C. A. Carver, R. A. Howard and W. D. Lane, Addressing different learning styles through course hypermedia, IEEE Transactions on Education, 42, 1999, pp. 33-38.

[6] J. Kuljis and F. Liu, A comparison of learning style theories on the suitability for elearning, Proceedings of the Conference on Web Technologies, Applications, and Services, ACTA Press, 2005, pp. 191-197.

[7] L. A. Richards-Ward, Investigating the relationship between two approaches to verbal information processing in working memory: An examination of the construct of working memory coupled with an investigation of meta-working memory, Massey University, Palmerston North, New Zealand, 1996.

[8] G. A. Miller, The magic number seven, plus or minus two: Some limit of our capacity for processing information, Psychology Review, 63, 1956, pp. 81-96.

[9] S. Graf, T. Lin and Kinshuk, The relationship between learning styles and cognitive traits - Getting additional information for improving student modelling, International Journal on Computers in Human Behavior, in press.

[10] R. M. Felder and B. A. Soloman, 1997. Index of Learning Style questionnaire. Retrieved 22 February, 2007, from http://www.engr.ncsu.edu/learningstyles/ ilsweb.html

[11] Web-OSPAN, 2007. Retrieved 22 February, 2007, from http://altrc.massey.ac.nz/ tylin/webOSPAN

[12] M. L. Turner and R. W. Engle, Is working memory capacity task dependent? Journal of Memory and Language, 28, 1989, pp. 127-154.

[13] W. de Neys, G. d'Ydewalle, W. Schaeken and G. Vos, A Dutch, computerized, and group administrable adaptation of the operation span test, Psychologica Belgica, 42, 2002, pp. 177-190.

[14] R. M. Felder and J. Spurlin, Applications, reliability and validity of the Index of Learning Styles, International Journal on Engineering Education, 21, 2005, pp. 103112. 